\title{
Fabrication of Graphene Field-Effect Transistor with Field Controlling Electrodes to Improve $f_{T}$
}

\author{
C. Al-Amin, M. Karabiyik and N. Pala
}

\begin{abstract}
In this letter, we report on the fabrication and DC/RF characterization of a novel Graphene Field-effect Transistor (GFET) with two additional contacts at the access regions. The additional contacts-Field Controlling Electrodes (FCEs), are capacitively coupled to the ungated access regions and independently biased to control the access resistance. The reduced access resistance resulted in an increased current-gain cutoff frequency $\left(f_{T}\right)$. The fabricated proposed device could be used for radio frequency (RF) applications.
\end{abstract}

Keywords: Graphene, Field Effect Transistor, RF, Fabrication, Access Resistance

Introduction: The advantages of Graphene as a channel material of FETs include but not limited to its perfect 2D confinement of carriers, high carrier mobility, mechanical flexibility, extremely high thermal conductivity and long phonon mean free path [1,2]. As Graphene is a zero bandgap material, there is no separation or forbidden states between Graphene's valence band and conduction band. In case of a conventional semiconductor material with nonzero bandgap (e.g. Silicon with a bandgap of $1.1 \mathrm{eV}$ ), electrons at the valence band need to gain energy equal to or more than the material bandgap to jump up to conduction band, thus contributing in electrical conduction. For transistors made of these materials, it is possible to cease the electron flow from valence band to conduction band by applying suitable gate bias, thus ceasing the channel current. The negligible current still passing through the channel in this condition is termed as off-state current. This small off-state current makes the on/off current ration of these devices very high which is important for reliable switching in logic devices. In contrast, due to the lack of bandgap in Graphene, electrons in its valence band can easily go to conduction band, thus contributing in conduction, and its cessation is not possible by applying gate bias. There is a non-zero minimum conduction point in Graphene FETs which can be considered as off-state current. However, this off state current is non-negligible, thus makes the device on/off current ratio unacceptably low for switching in logic devices [3]. On the contrary, significant advancement has been made on graphene devices for analog applications. The access resistance reduces the transconductance and drain current of FET, in general, and its adverse effect on current-gain, as well as, on current-gain cut-off frequency $\left(f_{T}\right)$ is well known. That is also true for GFET. To utilize the complete advantage of Graphene as a FET channel material, the set of limitations on the high-frequency performance of GFETs, arisen from the access resistances must be eliminated. Improving high frequency performance by reducing access resistance in III-N HFET and GFET has been already reported [4, 5, and 6]. Two Capacitively coupled and independently biased contacts (Field Controlling Electrode, FCE) at the access region can reduce the access resistance of GFET and increase $f_{T}$, significantly. If additional bias is applied to the capacitively coupled additional contacts, it induces additional carriers at the access region of the device which effectively reduces the access resistance. The current gain cut-off frequency is inversely proportional to access resistance. Thus by applying additional bias to the additional contacts, the induced additional carriers at the access region effectively increase $f_{T}$. The $f_{T}$ improvement of GFETs due to these additional contacts has been estimated from numerical and analytical calculations in [7, 8]. Here we have fabricated the novel structure GFET with two additional contacts at the access region, measured its DC characteristics and current-gain, and compared with same geometry conventional GFET without FCEs.

\section{Theory:}

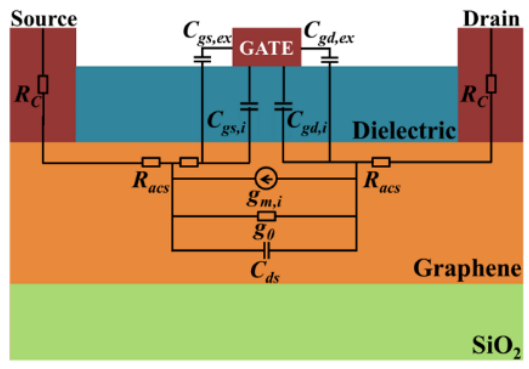

1

(C) 2016. This manuscript version is made available under the Elsevier user license http://www.elsevier.com/open-access/userlicense/1.0/ 
Fig. 1 Schematic of a conventional 3-terminal GFET on $\mathrm{SiO}_{2}$ with the small-signal equivalent circuit laid on top.

The schematic of a conventional 3-terminal GFET on $\mathrm{SiO}_{2}$ with the small-signal equivalent circuit laid on top is shown in Fig. 1. The time a carrier takes to travel from source to drain, termed as delay time, can be divided into parts: transit delay, $\tau_{\text {trans }}$ and parasitic delay, $\tau_{\text {par }}$. The transit delay is directly proportional to the intrinsic and extrinsic gate capacitance; whereas, the parasitic delay is proportional to the gate capacitances, as well as, on parasitic resistances. The current gain cut-off frequency $f_{T}$ is inversely proportional to the total delay time in the device and can be expressed as [9]

$$
1 / 2 \pi f_{T}=\tau_{\text {trans }}+\tau_{\text {par }}
$$

As show in [7, 8], the induced carriers caused by FCE modulation effectively reduces the resistance of the ungated access regions and thus results in a decrement of $\tau_{\text {par }}$ and increment of $f_{T}$.

Fabrication: Rather than following the conventional sequence of GFET fabrication steps starting with Graphene transfer and mesa etch, and ending with gate patterning, here we started the process by firstly pattering gate-FCE on substrate and finished by doing the mesa etch of Graphene, to prevent Graphene from excessive contamination due to exposure to resists, solvents, and developers. The $\mathrm{SiO}_{2}$ substrate was cleaned with acetone/isopropyl alcohol/methanol, rinsed with deionized (DI) water, and dried with $\mathrm{N}_{2}$ flow. $200 \mathrm{~nm}$ of 950 PMMA A4 was spin coated and oven baked at $185^{\circ} \mathrm{C}$ for 30 minutes. The Gate-FCE layer with alignment marks were patterned using ebeam lithography and developed in a solution of 1: 3 MIBK: IPA. 10-nm Ti/40-nm Au was evaporated using e-beam evaporator at a chamber pressure of $2 \times 10^{-7}$ mTorr and lifted-off in acetone, in a standard sonic bath. The next layer was the pads for which the patterned substrate was coated with $600 \mathrm{~nm} \mathrm{LOR} \mathrm{3B/600} \mathrm{nm} \mathrm{S1805} \mathrm{photoresist.} \mathrm{After}$ UV exposure, it was developed in developer MF-26A for 15 seconds, rinsed with DI, dried with $\mathrm{N}_{2}$, and a descum was done by oxygen plasma etching for 15 seconds. 10-nm Ti/500-nm Ni was evaporated for pad layer metallization and lifted-off. Following the Gate-FCE layer and pad layer formation, $20 \mathrm{~nm} \mathrm{HfO}_{2}$ was deposited by atomic layer deposition (ALD), as gate dielectric. Then it went through the same photolithographic process with mask to open windows for the pads. The $20 \mathrm{~nm}$ dielectric above the pads was etched by reactive ion etching (Chlorine + Argon) through the windows, followed by source/drain patterning. For the source/drain patterning, the same e-beam lithographic process was used and $10-\mathrm{nmTi} / 50-\mathrm{nm}$ Au was evaporated, followed by the same lift-off process in sonic bath. Source-Drain pad layer was patterned by the photolithographic process same as that used for Gate-FCE pad layer, followed by a 10-nm Ti/500-nm Au evaporation, and lift-off.

Single layer CVD Graphene grown on Copper sheet was coated with PMMA using a regular spin coating and was put floating on $\mathrm{FeCl}_{3}$ solution for 8 hours without any disturbance while copper surface touching the solution. Once the copper was completely etched, it was washed with DI water multiple times without flipping, and eventually was transferred on the patterned substrate. After transfer, the sample was put in a desiccant for 8 hours. Graphene covered with PMMA was then coated with S1805 photoresist and went through the same photolithographic process with mask once again, to open windows for mesa etch, through which oxygen plasma etching was used to etch PMMA and Graphene. Thus we prevented the Graphene from exposure to photoresist and developer. After mesa etch, the sample was put in acetone at $50^{\circ} \mathrm{C}$ for 15 minutes to remove PMMA over the Graphene channel.

Result and Discussion: The fabricated device had a gate length, $\mathrm{L}_{\mathrm{g}} \sim 2 \mu \mathrm{m}$, gate to source/drain access region length, $\mathrm{L}_{\mathrm{acs}} \sim 850 \mathrm{~nm}$, FCE length, $\mathrm{L}_{\mathrm{FCE}} \sim 270 \mathrm{~nm}$, FCE to gate length, $\mathrm{L}_{\mathrm{FCE}-\mathrm{g}} \sim 310 \mathrm{~nm}$, and FCE to source/drain length, $\mathrm{L}_{\mathrm{FCE}-\mathrm{s} / \mathrm{d}} \sim 270 \mathrm{~nm}$ as shown in Fig. 2. The device width was $80 \mu \mathrm{m}$. 

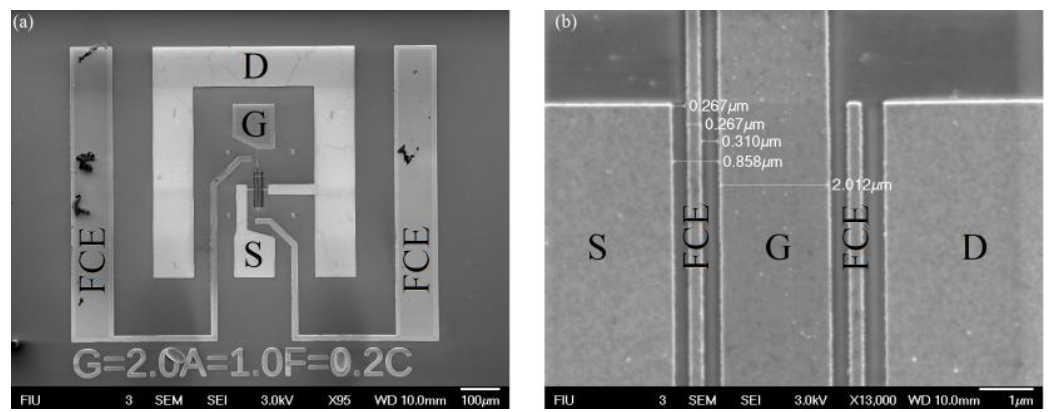

Fig. 2 (A) SEM image of the device at low magnification. (b) High magnification image with embedded scale showing FCE length, gate length, and access region length. The letters S, D, and G represent respectively the source, drain and gate terminal.

Graphene channel was characterized by Raman spectroscopy taken using a $532 \mathrm{~nm}$ laser with a spot size of $1 \mu \mathrm{m}^{2}$ As shown in Fig. 3. The 2D/G ratio (0.93) of the Raman spectrum confirms the presence of single layer graphene.

The DC characteristics of the fabricated device including drain voltage vs. drain current $\left(I_{d}-V_{d}\right)$ and gate voltage vs. drain current $\left(\mathrm{I}_{\mathrm{d}}-\mathrm{V}_{\mathrm{g}}\right)$ was measured using a semiconductor parameter analyzer. The drain current at a constant drain and gate voltage showed strong dependency on FCE bias and showed up to $10.1 \%$ increment at $\mathrm{V}_{\mathrm{FCE}}=-3.0 \mathrm{~V}$. The $I_{d}-V_{d}$ characteristics of the proposed GFET at $V_{F C E}=0$ is shown in Fig. 5 (b)

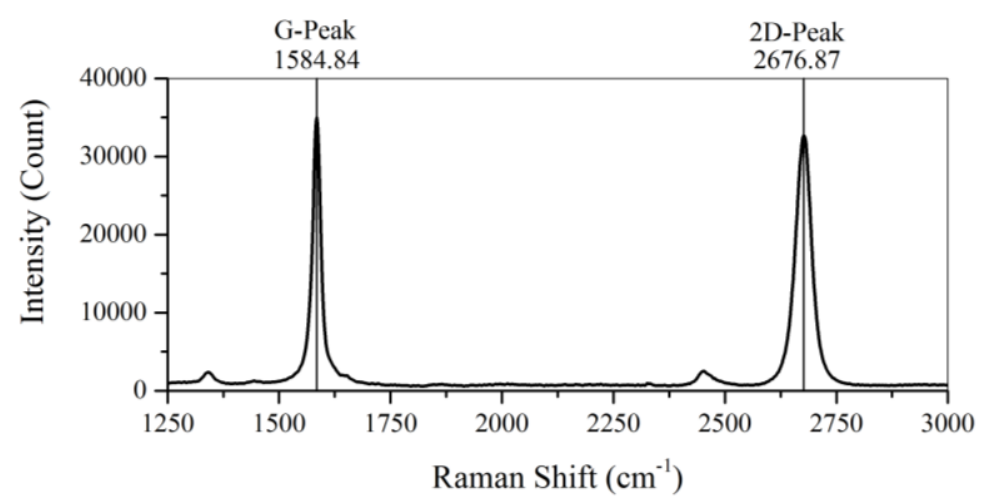

Fig. 3 The 2D/G ratio (0.93) of the Raman spectrum confirms the presence of single layer graphene forming the device channel.

The gate leakage current of the device is plotted in logarithmic scale and shown in Fig. 4 (a). The leakage current was at the order of a pA. In addition to gate leakage current, the FCE leakage current was measured as well. The FCE leakage current was at the order of pA as well, as shown in Fig. 4 (b).
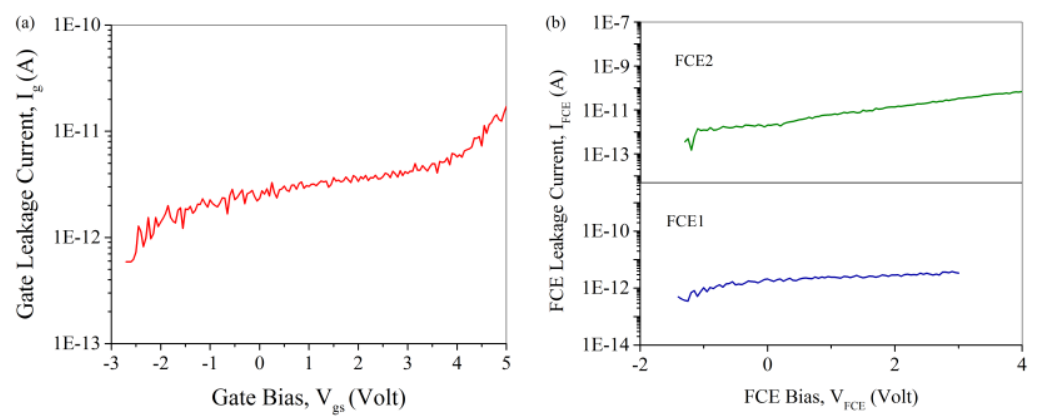

Fig. 4 (a) Gate leakage current (b) The FCE leakage current. 
The RF characteristics of the GFET was measured using a vector network analyzer integrated with a probe station and coplanar waveguide probes. Standard SOLT calibration was performed before measuring the 2-port SParameters, which were eventually converted to h21 parameter.
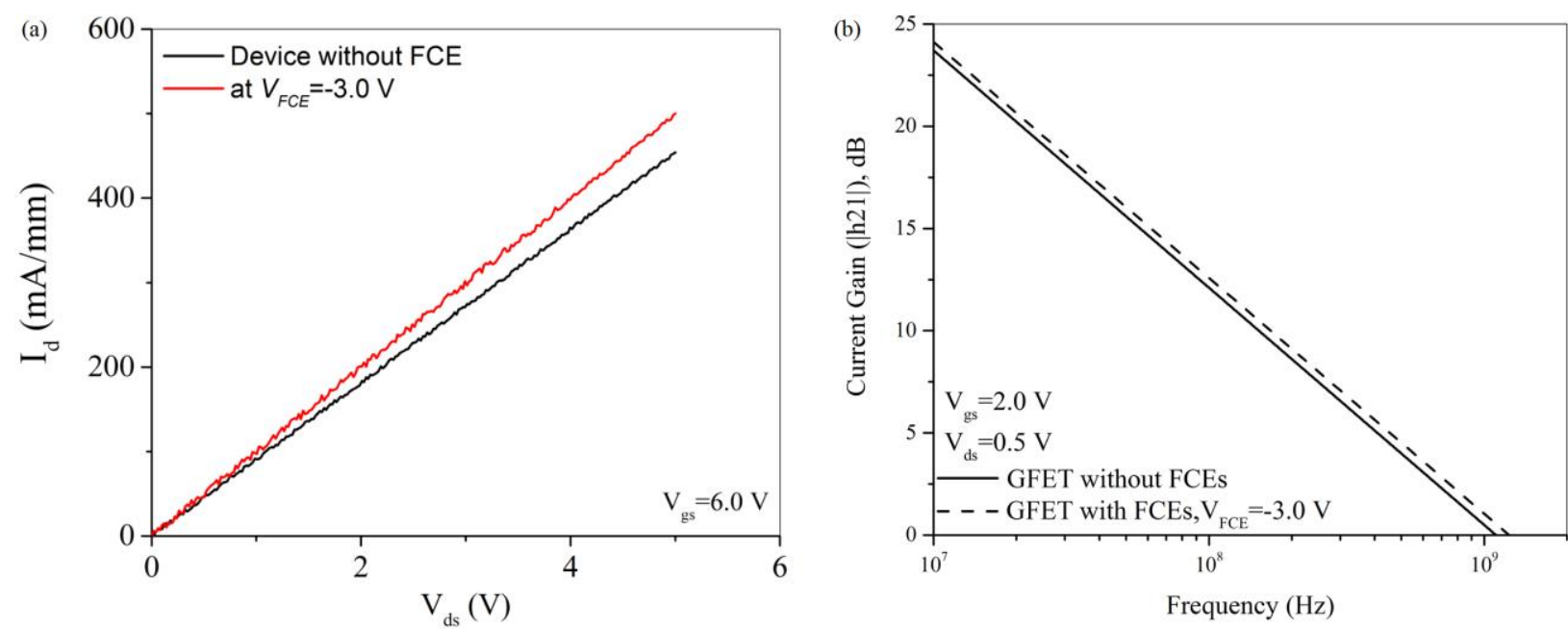

Fig. 5 (a) The $I_{d}-V_{d}$ characteristics of the conventional and proposed GFET $\left(V_{F C E}=-3.0 \mathrm{~V}\right)$, (b) Current gain of a conventional 3 terminal GFET along with that of a same geometry proposed GFET plotted with respect to frequency for comparison.

The current gain, |h21| is plotted with respect to frequency in Fig. 5 (b) for two different devices: conventional 3 terminal GFET and the same geometry GFET with proposed additional contacts (FCEs). The DC biasing condition at source, drain and gate were same for both of the devices whereas the proposed device had both of its FCEs biased at $-3.0 \mathrm{~V}$. From Fig. 5 (b), it is evident that $-3.0 \mathrm{~V}$ applied at both of the FCEs increases the current gain cut-off frequency from $1.10 \mathrm{GHz}$ to $1.22 \mathrm{GHz}$.

Conclusion: In conclusion, we experimentally demonstrated the superior RF performance of a proposed GFET with independently biased additional contacts at the access region, over a conventional GFET. The increment of current gain cut-off frequency was $10.9 \%$ and the proposed device could be used for high frequency applications.

Acknowledgments: This work is supported by NSF CAREER program with the Award Number: 0955013 and Army Research Office (Grant No. W911NF-12-1-0071). Chowdhury Al-Amin gratefully acknowledges the financial support provided by the FIU Graduate School Dissertation Year Fellowship.

C. Al-Amin, M. Karabiyik and N. Pala (Department of Electrical and Computer Engineering, Florida International University, Miami, FL 33174, USA)

E-mail: calam003@fiu.edu

\section{References}

1. Bolotin, K.I., Sikes, K., Jiang, Z., Klima, M., Fudenberg, G., Hone, J., Kim, P., Stormer, H.L.: 'Ultrahigh electron mobility in suspended graphene', Solid State Commun., 2008, 146, (9), pp. 351-355, doi:10.1016/j.ssc.2008.02.024

2. Balandin., A.A., Ghosh, S., Bao, W., Calizo, I., Teweldebrhan, D., Miao, F., Lau, C.N.: 'Superior thermal conductivity of single-layer graphene', Nano lett., 2008, 8, (3), pp. 902-907, doi: 10.1021/n10731872

3. Berrada, S., Nguyen, V.H., Querlioz, D., Saint-Martin, J., Alarcón, A., Chassat, C., Bournel, A., Dollfus, P.: 'Graphene nanomesh transistor with high on/off ratio and good saturation behavior', Applied Phys. Lett., 2013, 103, (18), pp. 183509-1-183509-5. doi: 10.1063/1.4828496 
4. Simin, G., Shur, M.S., Gaska, R.: '5-terminal THz GaN based transistor with field-and space-charge control electrodes', Int. J. High Speed Electron Syst., 2009, 19, (01). pp. 7-14, doi: 10.1142/S0129156409006047

5. Lin, Y., Farmer, D.B., Jenkins, K.A., Wu, Y., Tedesco, J.L., Myers-Ward, R.L., Eddy, C.R., Gaskill, D.K., Dimitrakopoulos, C., Avouris, P.: 'Enhanced performance in epitaxial graphene FETs with optimized channel morphology', IEEE Electron Device Letters, 2011, 32 (10), pp. 1343-1345, doi: 10.1109/LED.2011.2162934

6. Movva, H.C., Ramón, M.E., Corbet, C.M., Sonde, S., Chowdhury, S.F., Carpenter, G., Tutuc, E., Banerjee, S.: 'Self-aligned graphene field-effect transistors with polyethyleneimine doped source/drain access regions', Appl. Phys. Lett., 2012, 101, (18), pp. 183113-1-183113-4, doi: 10.1063/1.4765658

7. Al-Amin, C., Vabbina, P.K., Karabiyik, M., Sinha, R., Pala, N., Choi, W.: 'Improving High-Frequency Characteristics of Graphene FETs by Field-Controlling Electrodes', IEEE Electron Dev. Lett., 2013, 34, (9), pp. 1193-1195, doi: 10.1109/LED.2013.2272071

8. Al-Amin, C., Karabiyik, M., Vabbina, P., Sinha, R., Pala, N.: 'Field controlled RF Graphene FETs with improved high frequency performance', Solid-State Electronics, 2014, 95, pp. 36-41, doi: 10.1016/j.sse.2014.03.003

9. Wang, H., Hsu, A., Seup, L.D., Kang, K.K., Kong, J., Palacios, T.: 'Delay Analysis of Graphene Field-Effect Transistors', IEEE Electron Device Lett., 2012, 33, (3), pp. 324-326, doi: 10.1109/LED.2011.2180886 